. $B$ Abdi Khaireh, ${ }^{1}$ A Ahmed Abdi, ${ }^{3} \mathrm{~A}$ Bareh Matan, ${ }^{4} \mathrm{~S}$ Dao. ${ }^{1}$ Armed Forces Health Service, Djibouti, Djibouti; ${ }^{2}$ Medical Research Institute, Djibouti, Djibouti; ${ }^{3}$ Ministry of Health, Paul Faure Hospital, Djibouti, Djibouti; " 4 Faculty of Medecine, Bamako, Bamako, Mali

Background This work was done to study the HIV-TB co-infection at Paul FAURE Republic of Djibouti were tuberculosis is highly endemic. Our objectives was to identify the average profile of individuals concerned by co-infection in PAUL FAURE Hospital and the differences between two period of time. First period: January 2003 to April 2007 and second period: May 2007 to May 2008.

Methods The status of HIV-TB co-infection was studied through the distribution of HIV-TB co-infected patients. These patients were followed at PAUL FAURE Hospital.

This distribution was examined under thirteen parameters that were clinical, sociological and epidemiological over two distinct periods of time. The essential criterion for inclusion in the study was to be HIV-TB co-infected. The study included 104 cases of the 1 st period and 85 cases of 2 nd period.

Results The average profile of HIV-TB co-infected patient who was monitored at PAUL FAURE Hospital over the 1st period was: a man, between 26-45 years of age, Djiboutian, married, with modest income, moderately educated, weakly informed about HIV-TB diseases, having a TPM+ as a clinical form of TB with $12.50 \%$ chance of dying while receiving treatment, with survival rate at 1 year under ART equaling $19.44 \%$.

In the 2 nd period, the average profile was: a woman, between 26-40 years of age, Ethiopian, divorced, with modest income, not educated, not informed about HIV-TB diseases, drug users (Khat), a resident of Arhiba or Q4, having a TPM+ as clinical form of TB, being cured or still under treatment, with survival rate at 1 year under ART equaling $97.14 \%$.

Conclusions improving care and better monitoring of patients, as it was the case in second period, with systematic updating of sociological, clinical and epidemiological data can lead to a better management of the co-infection within the country.

\section{P3.339 HPV TYPE-SPECIFIC RISKS FOR HIGH-GRADE LESIONS: LONG-TERM FOLLOW-UP OF THE SWEDESCREEN STUDY}

doi:10.1136/sextrans-2013-051184.0792

V Smelov, M Elfström, J Dillner, the SwedeScreen study group. Karolinska Institutet, Stockholm, Sweden

Background The knowledge on HPV type-specific long-term absolute risks (AR) and population attributable proportions (PAR) for CIN2 + is limited. With the SwedeScreen population-based randomised controlled trial the effect of HPV testing in primary screening was evaluated.

Methods Overall 12,527 women (32-38 years) were randomised $1: 1$ to the intervention (cytology, HPV testing) and control arm (cytology, no action on HPV tests). Registry-based follow-up for cytological and histological test results was done (1997-2011). Type-specific ARs and PARs of CIN2+ were calculated. Poisson regression estimated the relative risk (RR) of new CIN2+. Multivariate analysis adjusted for co-infections. Women were censored at date of first CIN2 + or last registered cytology.

Results Over the entire follow-up, the joint PAR for 14 HR-HPV types was similar in the intervention and control arms $(69.3 \%$ versus $68.1 \%$ ). AR, RR and PAR were highest during the first screening round but risks were high throughout follow-up. HR-HPV + women developed CIN2+: $1-3$ years $13.6 \%$, 3-6 years $6.4 \%$, later $4.5 \%$. RRs: 89.5, 37.9, 12.2 and 9.0 during the first, second, third screening rounds and for $>9$ years of follow-up. Different HPV types tended to confer different risks over time: HPV18 increased, HPV16 and HPV31 stable, and others decreased. The HR types clustered in a highest, medium and a low AR groups (HPV16/18/31/33: 31-42\%,
HPV35/45/52/56/58: 13.8-24.8\%, HPV39/51/59/66/68: AR < 11\%). HPV16 contributed to the greatest proportion of CIN2+ in the population (first round PAR 38.8\%), followed by HPV52 (9.6\%), HPV31 (7.0\%) HPV18 (5.9\%) and HPV45 (5.2\%).

Conclusion HPV screening had minimal effect on the proportion of CIN2+ lesions caused by the HPV types screened for. HR-HPVassociated risks for CIN2+ continue to be strongly elevated over long-term (9-14 years) follow-up, particularly for HPV16, 18, 31 and particularly for CIN3+ lesions. The seven HR-HPV types 16/18/31/33/45/52/58 cause $73.9 \%$ of CIN2 + lesions. All 14 HR types cause $86.9 \%$.

\section{P3.340 STUDY OF KNOWLEDGE AND ATTITUDES OF MARRIED WOMEN AGED 10 TO 49 REFERRED TO HEALTH CENTRES AFFILIATED TO ISFAHAN UNIVERSITY OF MEDICAL SCIENCES AND HEALTH PROVIDERS EMPLOYED AT THESE CENTRES ABOUT AIDS AND SOME FACTORS RELATED TO -2011}

doi:10.1136/sextrans-2013-051184.0793

'M Meshkati, 'M Mostajeran, ${ }^{2} \mathrm{~A}$ Hajariyan, 'S Nematolahi, 'L Hosseyni. 'Province health centre, Isfahan, Iran; ${ }^{2}$ health centre, Isfahan, Iran

Background Expanding of AIDS is such an important issue that one of the main goal of WHO is increasing of knowledge about HIV in general population. Thus in forth developing programme of IRAN, prevention programmes for HIV has been mentioned. Education is necessary for changing of high risk behaviours.

Methods This descriptive study was conducted in 1390. The samples were 9207 married women between 10-49 years old who were coming to health centres and selected by systematic method and 2465 health providers were working in these centres.

Results Most of them were housewife (93.4\%) $55.5 \%$ of women had trained about HIV/AIDS but $86.6 \%$ of women don't have complete awareness about HIV/AIDS and just $13.4 \%$ of them have complete awareness. The first source for getting information was TV and then health care providers.there was a significant relation between the score of knowledge and their age, educational level and career $(p<0.001)$. the least knowledge was about transmitted and nontransmitted ways. Post counselling was given to $22.4 \%$ tested women. The mean score for attitude was $84.3 \pm 11.2$ and was observed statistical significant between the score of attitude and their age, educational level, addressing, HIV testing and career $(\mathrm{p}<0.001)$.for health care provider there wasn't a significant relation between the score of knowledge and their age and career. The mean score for attitude was $91.1 \pm 7.5$ and there was observed statistical significant between the score of attitude and their educational level and career $(\mathrm{p}<0.001)$.

Conclution Findings showed limited knowledge about HIV so we need to improve methods of training and use variety in our awareness programme such as peer groups. It is necessary to train health providers about VCT. In addition we must try for decreasing stigma, keeping the rights of patients and increasing social marketing for VCT, care and treatment of people live with HIV/AIDS

\section{P3.341 SEX AND THE LONDON OLYMPICS 2012 PART 1. IMPACT ON COMMUNITY SEXUAL HEALTH SERVICE PROVISION IN LONDON}

doi:10.1136/sextrans-2013-051184.0794

${ }^{1} \mathbf{G}$ Brook, ${ }^{2} \mathrm{~J}$ Cassell, ${ }^{3} \mathrm{C}$ Mercer, ${ }^{4} \mathrm{~K}$ Coyne, ${ }^{5} \mathrm{H}$ Maguire, ${ }^{5} \mathrm{G}$ Hughes, ${ }^{5} \mathrm{P}$ Crook. ${ }^{1} \mathrm{Central}$ Middlesex Hospital, London, UK; ${ }^{2 B}$ Brighton and Sussex Medical School, Brighton, UK; ${ }^{3}$ University College, London, UK; ${ }^{4}$ Homerton University Hospital, London, UK; ${ }^{5}$ Health Protection Agency, London, UK 